\title{
RecombinantActivated Factor VII, as a Remedy for Intractable Coagulopathy Following Massive Transfusion in a Case of Giant Cranial Neurofibroma
}

\author{
Keyvan Tayebi Meybodi ${ }^{1}$; Mohammad Taghvaei ${ }^{1}$; Nazi Derakhshanrad ${ }^{2}$; Zahid Hussain \\ Khan $^{2,3}$; Hooshang Saberi ${ }^{1,2,}$ \\ ${ }^{1}$ Department of Neurosurgery, Imam Khomeini Hospital, Tehran University of Medical Sciences, Tehran, IR Iran \\ ${ }_{2}^{2}$ Brain and Spinal Injuries Research Center, Imam Khomeini Hospital, Tehran University of Medical Sciences, Tehran, IR Iran \\ ${ }^{3}$ Department of Neuroanesthesiology, Imam Khomeini Hospital, Tehran University of Medical Sciences, Tehran, IR Iran \\ ${ }^{*}$ Corresponding author: Hooshang Saberi, Brain and Spinal Injuries Research Center, Imam Khomeini Hospital Complex, Keshavarz Boulevard, P. O. Box: 14185-61, Tehran, IR Iran. Tel: \\ +98-2166581560, Fax: +98-2166938885, E-mail: hgsaberi@yahoo.com
}

Received: February 18, 2014; Revised: Mar 17, 2014; Accepted: Mar 20, 2014

\begin{abstract}
Introduction: Neurosurgical interventions have been associated with troublesome bleeding during and after the procedures. There are cases of intractable coagulopathy with life threatening bleeding. In this study, we reported a patient with neurofibromatosis type 1 , and huge cranial neurofibroma, associated with massive bleeding after excision.

Case Presentation: A 22 year-old man was referred to this center with facial deformity due to a large craniofacial neurofibroma. He underwent craniotomy and tumor resection, but intraoperative blood loss was massive, and epidural hematomata developed at the operative site due to coagulopathy. The drains still were draining fresh blood and the patient was severely anemic. After consultation with a hematologist, rFVIIa was administered intravenously to successfully control the bleeding.

Conclusions: While using recombinant rFVIIa is life-saving, it should not be a substitute for life saving urgent surgeries and should be cautiously used as a last resort in conjunction with conventional medico-surgical care.
\end{abstract}

Keywords:Recombinant activated factor VII; Coagulopathy; Intracranial Hemorrhages

\section{Introduction}

Neurosurgical interventions have been associated with troublesome bleeding during and after the procedures. Postoperative hematoma and massive transfusion are well-known dilemmas after giant cranial tumor removal, sometime very severe (1). Classic treatment modalities for postoperative coagulopathy include fresh frozen plasma (FFP), epsilon aminocaproic acid, calcium gluconate, prothrombin complex concentrate (PCC), tranexamic acid, and fresh whole blood. Nevertheless, there are cases of intractable coagulopathy with life threatening bleeding (2). In this study, we reported a patient with neurofibromatosis type 1 , and huge cranial neurofibroma, associated with massive bleeding after excision, for whom, recombinant activated Factor VII (rFVIIa) was used to stop life threatening and troublesome bleeding.

\section{Case Presentation}

A 22 year-old man was referred to this center with facial deformity due to a large craniofacial neurofibroma. He had undergone surgical interventions seven times since age seven. The last surgery was 6 years before this admission. At previous surgeries, the patient had undergone frontoparietotemporal craniectomy at the tumor site. The tumor had remodeled the underlying cranium and a large redundant tumoral skin was present from the upper to the lower face (Figure 1). He was medically normal, and the coagulation profile had no abnormality. There was no preoperative medication except that the patient was taking phenytoin as an anticonvulsant. The preoperative MRI scans are presented (Figure 2). At surgery, the previous incision was opened (harmonious with bicoronal line). The tumor was attached to cranium and had infiltrated the skin, and therefore was shaved from the skin. The craniectomy was enlarged, and dura mater was detached from the tumor, where it was adherent to the dura through the previous craniectomy site. The redundant dura mater evolved from expansion of the cranium, was trimmed, and the dura was closed. A titanium mesh was molded to the shape and size of craniectomy and placed over the bony defect. Subgaleal drain was placed, and the skin was closed. Because of excessive blood loss during the operation, blood products including packed

Implication for health policy/practice/research/medical education:

Neurosurgical interventions have been associated with troublesome bleeding during and after the procedures. Postoperative hematoma and massive transfusion are well-known dilemmas after giant cranial tumor removal, sometime very severe. In this study, we reported a patient with neurofibromatosis type 1, and huge cranial neurofibroma, associated with massive bleeding after excision, for whom, recombinant activated Factor VII (rFVIIa) was administered to stop life threatening and troublesome bleeding.

Copyright (c) 2015, Tehran University of Medical Sciences. This is an open-access article distributed under the terms of the Creative Commons Attribution License, which permits unrestricted use, distribution, and reproduction in any medium, provided the original work is properly cited. 
Tayebi Meybodi Ket al.

Figure 1. Multislice Computed Tomography With 3d Recontrction and Surface Rendering
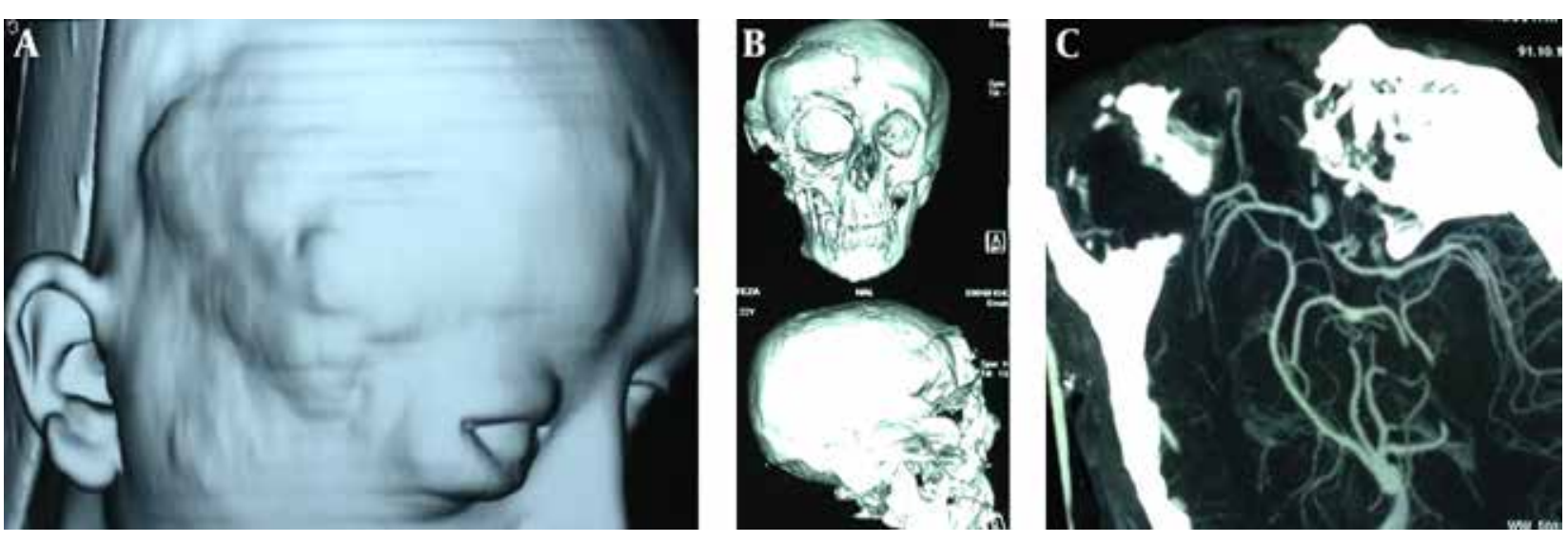

A. surface rendered multislice computed tomography of the patient's cranial tumor invading the upper and mid-facial regions. B. 3D reconstruction of computed tomography of craniofacial skeleton, indicating a large bone defect at the right fronto-orbito-maxillary bony structures. C. Computed tomographic cerebral angiography showing the right vasculature including middle and anterior cerebra arteries herniating through the bony skull defect. The tumor is supplied totally by the external carotid system.

Figure 2. Preoperative Cranial MRI With T1 and T2 Pulse Sequences
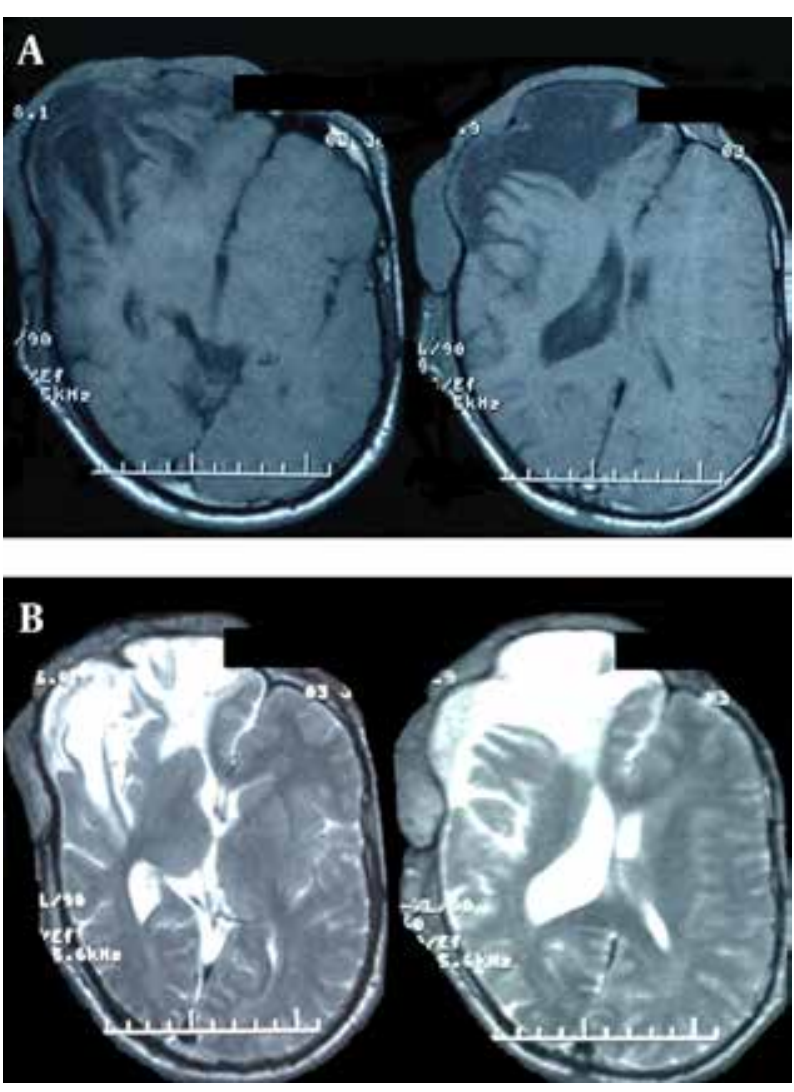

A. T1-weigted images of craniofacial mass lesion showing low signal intensity brain malacia of previous surgeries as well as solid portions of extradural tumor. B. T2-weighted images of the same region, showing a high signal cyst formation intradurally, as well as large extradural isointense mass lesion, invading the subcutaneous tissue.
Figure 3. Urgent Computed Tomogram Just Before Evacuation of Isodense Huge Extradural Right-sided Frontal Hematoma

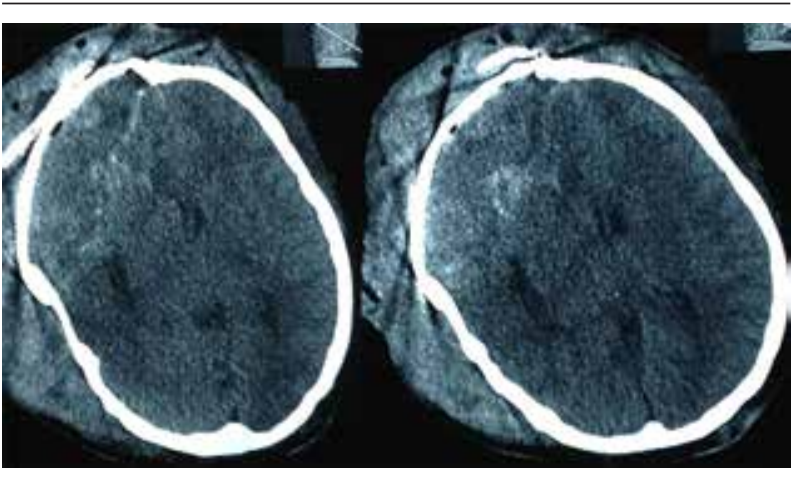

Note that the density of hematoma is not high due to hyper-acute timing and low hemoglobin content. Titanium mesh covers the hematoma. In addition, the basal perimesencephalic cisterns are obliterated.

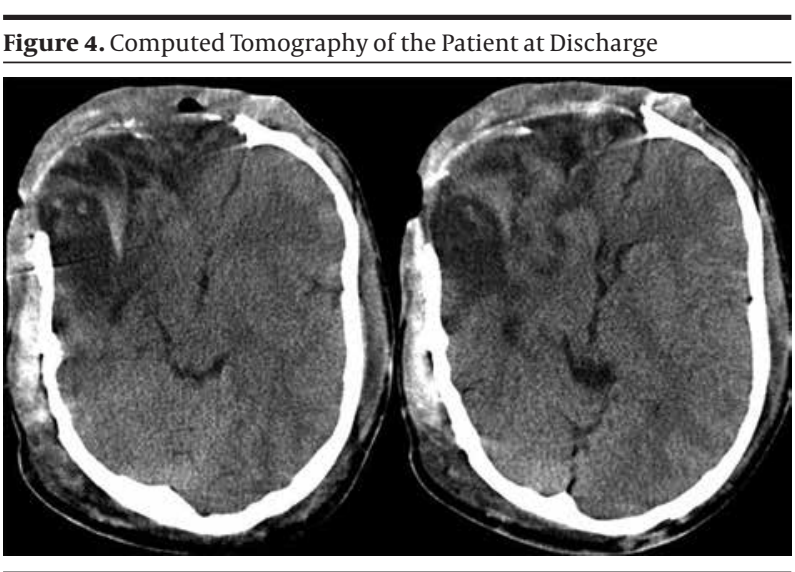

Note low density in the right fontal region, corresponding to the preoperative brain malacia, scattered contusions. The basal cisterns are open. 
red blood cell, platelet, and fresh frozen plasma were given, and monitoring of hematocrit and coagulation profiles was performed multiple times daily. Postoperatively, the patient was transferred to ICU for close monitoring, while he was intubated. Upon arrival, hypotension and tachycardia were detected, and a stat of vasopressor was administered. A few hours later, because of severe blood loss through the drain, decreased hematocrit, and a rapid decline in blood pressure (hypovolemic shock), multiple packs of red blood cell, platelets, and fresh frozen plasma were administered. Eighteen hours after the operation, the patient developed loss of consciousness, and unilateral dilated pupil (concordant with the site of operation). With the assumption of acute epidural hematoma (Figure 3), he was transferred to the operating room and the incision was opened. The titanium mesh was removed and epidural hematoma was evacuated. Dura was tacked up and multiple drains were placed. Soon after surgery, he was alert and oriented, but blood loss was extensive through the drains. Slightly abnormal prothrombin and partial thromboplastin time were detected, which were presumably due to multiple transfusions of packed red blood cells. These abnormalities were corrected with infusion of FFP and platelets. Despite the correction of coagulation profile, bleeding was not ceased. After consultation with a hematologist, rFVIIa was administered at a dose of $4 \mathrm{mg}(80 \mu \mathrm{g} / \mathrm{kg})$, intravenously. The bleeding was reduced and the patient's need for blood products was decreased. A few hours later, bleeding was increased to its level before the administration of activated factor VII, therefore a $6 \mathrm{mg}(120 \mu \mathrm{g} / \mathrm{kg})$ dose of the drug was given intravenously. The bleeding was totally controlled. Close monitoring of coagulation profile and hematocrit was performed, and any abnormality was aggressively treated. There was no new onset bleeding thereafter. The drains were removed two days later. The patient was discharged 12 days after the operation (Figure 4) without any neurological deficit and bleeding problem.

\section{Discussion}

Coagulopathy may be observed before neurosurgical interventions because of underlying medical problems and/or during the management of hypervascular tumors, or extensive craniofacial surgeries, due to severe blood loss and massive transfusion. Activated factor VII has been used for such complicated situations as a last resort. Recombinant factor VIIa was administered for the treatment of intracerebral hemorrhage in fifteen patients with no adverse thromboembolic complication (3). In urgent neurosurgical intervention for patients with non-hemophilic preexisting hemophilia, rFVIIa has been used safely without major hemorrhagic complications in 9 patients. There were no thromboembolic complica- tions (4). In our case, there was no evidence of thromboembolic phenomenon.

In pediatric neurosurgery, this drug has been used for craniofacial reconstruction and malignant tumors at a dose of $100 \mu \mathrm{g} / \mathrm{kg}$ intravenously (5). The drug was used for the first time for acute subdural hematoma in 2002 (6). The risk of arterial thromboembolism has been reported as 1.4-1.9\% (2). Besides, it has been used to prevent rebleeding in acute subarachnoid hemorrhage. The drug has been used for traumatic intracranial hematoma at risk of expansion, without any coagulopathy and the authors have concluded that, it may prevent hematoma enlargement (7). In our case, prevention of hematoma expansion was achieved after surgery in the presence of severe coagulopathy, and our study does not support the use of this drug in non-urgent normocoagulative hemorrhagic state.

The most commonly therapeutic dosage has been 90-120 $\mu \mathrm{g} / \mathrm{kg}$, while hemorrhage prevention has been achieved with as low as $5 \mu \mathrm{g} / \mathrm{kg}$ (8). The medium doses $(10-40 \mu \mathrm{g} / \mathrm{kg})$ have been used in association with FFP and vitamin $\mathrm{k}(9)$ and higher doses (120-160 $\mu \mathrm{g} / \mathrm{kg}$ ) may be associated with arterial thromboembolism (10). In our case, the dose was $80 \mu \mathrm{g} / \mathrm{kg}$, which was further raised to $120 \mu \mathrm{g} / \mathrm{kg}$. The high cost of rFVIIa may preclude clinical application in some centers, however further studies may be needed for a decision protocol for local third party payers (11).

A case presented by Khan et al (12) indicated that although hypercoagulable complications following rFVIIa infusion are rare, but they do occur and thus all patients receiving rFVIIa should be carefully monitored for thromboembolic phenomenon which if occurs can be fatal. Recombinant factor VIIa has been approved for patients with hemophilia (13), but it is off-label use in other situations where the surgeon is at a crossroad in ligating or else coagulating active bleeders is not to be recommended. Finally, the use of recombinant rFVIIa should not be a substitute for life saving urgent surgeries and should be cautiously used as a last resort in conjunction with conventional medico-surgical care.

\section{Acknowledgements}

We are indebted to our patient for permission to publish this case report, and Dr. Shahverdi and Dr. Gholamreza Tugeh for their supports.

\section{Authors' Contributions}

All the authors substantially contributed to preparing the paper.

\section{References}

1. Gerlach R, Tolle F, Raabe A, Zimmermann M, Siegemund A, Seifert $\mathrm{V}$. Increased risk for postoperative hemorrhage after intracranial surgery in patients with decreased factor XIII activity: implications of a prospective study. Stroke. 2002;33(6):1618-23. 
2. Beshay JE, Morgan H, Madden C, Yu W, Sarode R. Emergency reversal of anticoagulation and antiplatelet therapies in neurosurgical patients. J Neurosurg. 2010;112(2):307-18.

3. Sutherland CS, Hill MD, Kaufmann AM, Silvaggio JA, Demchuk AM, Sutherland GR. Recombinant factor VIIa plus surgery for intracerebral hemorrhage. Can J Neurol Sci. 2008;35(5):567-72.

4. Park P, Fewel ME, Garton HJ, Thompson BG, Hoff JT. Recombinant activated factor VII for the rapid correction of coagulopathy in nonhemophilic neurosurgical patients. Neurosurgery. 2003;53(1):34-8.

5. Uhrig L, Blanot S, Baugnon T, Orliaguet G, Carli PA, Meyer PG. Use of recombinant activated factor VII in intractable bleeding during pediatric neurosurgical procedures. Pediatr Crit Care Med. 2007;8(6):576-9.

6. Veshchev I, Elran H, Salame K. Recombinant coagulation factor VIIa for rapid preoperative correction of warfarin-related coagulopathy in patients with acute subdural hematoma. Med Sci Monit. 2002;8(12):CS98-100.

7. Mayer SA, Brun NC, Begtrup K, Broderick J, Davis S, Diringer $M N$, et al. Efficacy and safety of recombinant activated factor VII for acute intracerebral hemorrhage. $N$ Engl $\mathrm{J} \mathrm{Med.}$ 2008;358(20):2127-37.

8. Pickard JD, Kirkpatrick PJ, Melsen T, Andreasen RB, Gelling L,
Fryer T, et al. Potential role of NovoSeven in the prevention of rebleeding following aneurysmal subarachnoid haemorrhage. Blood Coagul Fibrinolysis. 2000;11 Suppl 1:S117-20.

9. Sorensen B, Johansen P, Nielsen GL, Sorensen JC, Ingerslev J. Reversal of the International Normalized Ratio with recombinant activated factor VII in central nervous system bleeding during warfarin thromboprophylaxis: clinical and biochemical aspects. Blood Coagul Fibrinolysis. 2003;14(5):469-77.

10. Diringer MN, Skolnick BE, Mayer SA, Steiner T, Davis SM, Brun $\mathrm{NC}$, et al. Risk of thromboembolic events in controlled trials of rFVIIa in spontaneous intracerebral hemorrhage. Stroke. 2008;39(3):850-6.

11. Earnshaw SR, Joshi AV, Wilson MR, Rosand J. Cost-effectiveness of recombinant activated factor VII in the treatment of intracerebral hemorrhage. Stroke. 2006;37(11):2751-8.

12. Khan ZH, Soltani AE, Rahmani P. Postoperative pulmonary thromboembolism possibly associated with recombinant activated factor VII infusion for the treatment of uncontrolled hemorrhage during vertebral instrumentation. $J$ Anesth. 2007;21(2):258-60.

13. Kenet G, Martinowitz U. Single-dose recombinant activated factor VII therapy in hemophilia patients with inhibitors. Semin Hematol. 2008;45(2 Suppl1):S38-41. 